\title{
The effect of warning interval on signal detection and event-related slow potentials of the brain
}

\author{
N. E. LOVELESS \\ University of Dundee, Dundee DD1 $4 H N$, Scotland
}

\begin{abstract}
The amplitude of a slow potential wave in the EEG was examined during performance of a signal detection task under several fixed intervals between warning signal and critical signal. It was observed that the probability of detecting the critical signal decreased as the interval increased, and that this effect was due to a change in sensitivity rather than a change in criterion. The time-course of the change in sensitivity was related to that of the slow potential wave, which peaks shortly after the warning signal and then declines over a period of several seconds, and is interpreted as a component of the orienting response. It is suggested that the mechanism whose function is reflected in this wave also accounts for some effects of foreperiod duration and warning signal characteristics in reaction time tasks.
\end{abstract}

Since the initial report by Walter, Cooper, Aldridge, McCallum, and Winter (1964) that a slow negative wave (the "contingent negative variation," or $\mathrm{CNV}$ ) develops in the EEG during the foreperiod of a simple reaction task, a considerable volume of research has confirmed that similar potential shifts occur during performance of a variety of tasks in which the subject has to respond in some way to the second of a pair of stimuli, and that these shifts appear to be sensitive to a number of psychological variables (Tecce, 1972). It is, however, arguable that this body of evidence has not been related to the processes involved in task performance in such a way as to elucidate the functional significance of these event-related components of the EEG (Donchin, Otto, Gerbrandt, \& Pribram, 1971).

In a series of recent experiments, slow potential changes recorded during the foreperiod of a simple reaction task have been related to variations in preparatory set induced by manipulation of several variables. Loveless and Sanford (1974a) investigated the effects of temporal uncertainty, comparing a regular condition in which foreperiod was constant over a block of trials with an irregular condition in which foreperiods varied in duration from trial to trial. They confirmed the well-known behavioral effects that reaction time (RT) is shorter in the regular condition and increases with increasing foreperiod duration, and showed that these effects were reflected in the amplitude and time-course of the slow potentials. In the irregular condition, the main feature of the averaged waveform was a surface-negative wave peaking about 1 sec after the

This investigation was supported by a grant from the Science Research Council of the United Kingdom. The author is grateful for the technical assistance of Mr. B. L. Potter and Mrs. H. Fraser-Smith. warning signal, and then gradually declining in amplitude; this "O wave" was interpreted as part of the orienting response to the warning signal. In the regular condition, the $O$ wave was still present, but superimposed upon it was a further negative potential (E wave) which developed late in the foreperiod, anticipating the occurrence of the imperative signal and subsequent response. It thus appeared that variations in preparatory set related to predictability of the foreperiod were reflected in the $\mathrm{E}$ wave, while those related to foreperiod duration were reflected mainly in the terminal amplitude of the $O$ wave. This experiment therefore strongly suggested that the $\mathrm{CNV}$ is not a unitary phenomenon. A similar distinction between orienting and anticipatory potentials was made by Weerts and Lang (1973), who reported habituation of the orienting potential.

Subsequent experiments were designed to manipulate the two potentials separately and examine their possible links with the mechanisms determining RT. Loveless and Sanford (1974b) confirmed the classical finding that "motor set" instructions produce faster RTs than "sensory set" instructions, while "normal" instructions have an intermediate effect. Moreover, the effect of instructions upon RT interacted with imperative signal intensity in a manner compatible with models of simple RT (Grice, 1968; John, 1967; McGill, 1963) which suppose that evidence of the presence of the imperative signal accumulates at a rate proportional to its intensity until a critical level is reached at which the response is released. The inferred effects of instructions upon the criterion were reflected in $\mathrm{E}$-wave amplitude; $\mathrm{O}$-wave amplitude was not affected. It therefore seemed plausible to conclude that the development of the $E$ wave was a physiological concomitant of a lowering of response threshold at the anticipated time of imperative signal presentation. 
In contrast, Loveless and Sanford (1975), while confirming the tinding of Behar and Adams (1966) that increasing the intensity of the warning signal decreased RT, found that warning signal intensity was correlated with peak amplitude of the $O$ wave. This suggested that the warning signal affected performance through some mechanism other than regulation of the response threshold. It was proposed that warning signal intensity affects the rate at which evidence of the presence of the imperative signal is accumulated, thus altering its effective intensity. Moreover, since it was again found that RT increased with foreperiod duration, it was hypothesized that the sensitizing effect of the warning signal decreases with time, and that this effect might be reflected in the declining amplitude of the $\mathrm{O}$ wave.

The proposition that the $O$ wave is associated with a mechanism regulating perceptual sensitivity is compatible with the function of the orienting response particularly stressed by Sokolov (1963). namely facilitation of the detection of a subsequent stimulus. This hypothesis cannot be tested through further study of the simple reaction task, since existing models of this task do not permit a ready separation of sensitivity and criterion effects. A possible way out of this difficulty is to deternine whether comparable EEG effects are obtained in a situation which yields measures derived from signal detection theory. An obvious choice is a study of the effect of foreperiod duration upon signal detection, since this is known to be broadly consistent with its effect upon RT. A series of experiments (Howarth \& Treisman, 1958, 1961; Treisman \& Howarth, 1959) has shown that sensory threshold, as determined by traditional psychophysical measures, is inversely related to foreperiod duration, where this is regular over a block of trials. The authors considered two possible explanations of this effect: first, that the warning signal gives rise to an arousal reaction, which causes an immediate but decaying increase in sensitivity to following stimuli; second, that the subjective threshold is lowered at the time when the critical stimulus is expected, but that this strategy is affected by the increased difficulty of time estimation as foreperiod lengthens. A further investigation by Treisman (1964) provided positive support for the second hypothesis. It should be noted, however, that the two hypotheses are not mutually exclusive; and the findings of Loveless and Sanford (1974a) suggest that both effects may be present when the foreperiod is predictable.

Ideally, it would have been desirable to follow the general paradigm of the latter experiment by obtaining measures of signal detection and slow potential amplitude as functions of foreperiod duration in both regular and irregular series. This would test the prediction that, in the irregular series, only an $\mathrm{O}$ wave would be obtained in the averaged waveform and the behavioral differences between foreperiods would be manifested in sensitivity changes, as measured by $d^{\prime}$, whereas in the regular series, the $E$ wave would also be present, and the behavioral differences between series would be shown in criterion changes, as measured by $\beta$. Unfortunately, there appears to be no simple way of applying signal detection theory to an irregular series, since this requires some procedure for partitioning responses among possible times of signal presentation (Egan, Schulman, \& Greenberg, 1961b). It was therefore decided to confine the present experiment to regular series. It was argued that if an $E$ wave was obtained in addition to an $\mathrm{O}$ wave, the differences in their combined amplitude between foreperiods would nevertheless depend mainly on the $\mathrm{O}$ wave; and it was predicted that foreperiod variation would affect $d$ '.

\section{METHOD}

\section{Subjects}

The subjects were seven undergraduate students who were naive as to the purpose of the experiment. They were paid for their services at a fixed hourly rate.

\section{Procedure}

All stimuli were delivered through noise-excluding headphones (Koss PRO-4AA), and masking of extraneous sound was provided by a continuous white noise of about $40 \mathrm{~dB}$. The warning signal was a $1-\mathrm{kHz}$ tone of $70-\mathrm{dB}$ intensity lasting $100 \mathrm{msec}$. The critical stimulus was a burst of white noise lasting $100 \mathrm{msec}$ superimposed upon the background noise. An individual threshold level was determined for each subject by the method of limits and then used throughout the remainder of the experiment, which employed a yes-no procedure, the subject responding verbally. Trials were arranged in sets of 10 , with the constraint that each set contain 5 positive and 5 negative trials, but the sets constituting a block of trials were presented without a break. Four foreperiods were used, namely $0.5,1.0,3.0$, and $6.0 \mathrm{sec}$, these intervals being the same as those used in the RT experiment by Loveless and Sanford (1974a), except that a 15.0 -sec interval was omitted.

Each subject attended for three sessions of about $1 \mathrm{~h}$. The first was a practice session, consisting of a block of 30 trials at each foreperiod. The experiment proper used blocks of 100 trials, blocks for two foreperiods being presented at the second session and for the other two foreperiods at the third. The order of presentation of foreperiods was randomized between subjects. There were intervals of about $3 \mathrm{~min}$ between blocks and of about $10 \mathrm{sec}$ between trials. The intertrial interval was varied irregularly to avoid giving an additional time cue. Knowledge of results was not provided.

\section{Recording}

After cleaning the scalp with isopropyl alcohol, chlorided silver cup electrodes were applied with Neptic electrode gel at a resistance of 5.000 ohms or less. EEG was recorded from electrodes placed along the midline in supra-orbital, vertex. and occipital positions. Reference was to linked mastoids, except that the vertex electrode was referred to a potentiometer connected between the mastoid and supra-orbital electrodes and adjusted so as to minimize contamination from ocular potentials due to vertical eye movements. Ground electrodes were placed on the forehead. The EEG was amplified with a modified Elema-Schönander Mingograph Model 160 fitted with universal amplifiers. The high-frequency cutoff filter was set at $30 \mathrm{~Hz}$, and the time constant was $20 \mathrm{sec}$. except that a parallel dc recording was made from the vertex electrode. A 10 -sec epoch of analogue signal covering each 
trial was digitized by a $\mathrm{OEC}$ PDP-12A computer at a rate of 40 msec point and stored on magnetic tape for subsequent averaging.

Before averaging. waveforms from all three channels were carefully compared for evidence of contamination by artefacts, especially blinking, since the subtraction method used to compensate for vertical eye movements does not give complete protection against the large blink potential (Corby \& Kopell, 1972; Weerts \& Lang. 1973). Subjects had been instructed to maintain visual fixation on a target of concentric rings and to refrain from blinking during the period between warning signal and response. They were told that they were free to blink during the first few seconds after they had responded. These precautions were generally successful, but the data for one subject were totally discarded because of contanination by gross eye movements. For the remaining six subjects, it was necessary to exclude only a few trials on which blinks occurred during the foreperiod. Averaged waveforms of the remaining trials showed no evidence of ocular artefact.

The terminal amplitude of the slow potential developed at the vertex electrode during the foreperiod was measured as the difference between the mean amplitudes of the averaged EEG over a period of 500 msec preceding the warning signal and a period of $200 \mathrm{msec}$ preceding the critical stimulus. Measurements were made on the de recording.

\section{RESULTS}

Mean data for the six subjects whose records were usable are shown in Table 1. Criterion scores have been transformed to $\log \beta$ as a precaution against skewness of the distribution.

Analysis of variance applied to the behavioral data shows a significant effect of foreperiod duration upon $\mathrm{d}^{\prime}[\mathrm{F}(3.15)=5.18, \mathrm{p}<.05]$. The Tukey a test (Winer, 1970) shows that the values of $\mathrm{d}^{\prime}$ at the 0.5 -, $1.0-$, and $3.0-\mathrm{sec}$ intervals are significantly greater than the value at the 6.0 -sec interval $(\mathrm{p}<.05, .01$. and .05 , respectively). Although the mean value of $\log \beta$ across subjects decreases systematically as foreperiod lengthens, this pattern is not consistent between subjects, and the effect is not significant $[F(3.15)=2.57, \mathrm{p}>.05]$.

An important advantage of the signal detection paradigm is that it allows the EEG data to be segregated on both the stimulus condition and the behavioral response (Donchin \& Sutton, 1970; Sutton, 1969). In particular, Hillyard, Squires, Bauer, and Lindsay (1971) reported a difference in slow potential amplitude between the correct and incorrect responses of a signal detection experiment. The EEG data were therefore initially sorted according to stimulus-response contingencies and tested for a similar difference, but none was found. The probable reason for this is the relatively high detection rate in the present experiment, since Hillyard et al. (1971) noted that their finding held only for low performance levels and Paul and Sutton (1972) found no difference between categories with a moderate rate of detection. Amplitude measures for all categories were therefore pooled. Analysis of variance showed a significant effect of foreperiod duration on terminal amplitude $[F(3,15)=3.35$.
Table 1

Means of $d^{\prime}, \log \beta$, and Slow-Potential Amplitude as Functions of Foreperiod Duration

\begin{tabular}{|c|c|c|c|c|}
\hline & \multicolumn{4}{|c|}{ Foreperiod Duration (Sec) } \\
\hline & .5 & 1.0 & 3.0 & 6.0 \\
\hline $\mathrm{d}^{\prime}$ & 1.84 & 2.02 & 1.62 & 1.04 \\
\hline $\log \beta$ & .70 & .63 & .55 & .31 \\
\hline Amplitude $(\mu \mathrm{V})$ & -.6 & -6.5 & -3.2 & -2.1 \\
\hline
\end{tabular}

$p<.05]$. The Tukey test showed the terminal potential at the $1.0-\mathrm{sec}$ interval to be significantly higher (more negative) than at the 0.5 - and $6.0-\mathrm{sec}$ intervals.

Composite plots of vertex potentials, obtained by averaging across subjects, are shown in Figure 1. The plots have been truncated at the time of presentation of the critical stimulus, since the remainder of the record is not relevant to the hypothesis under test and
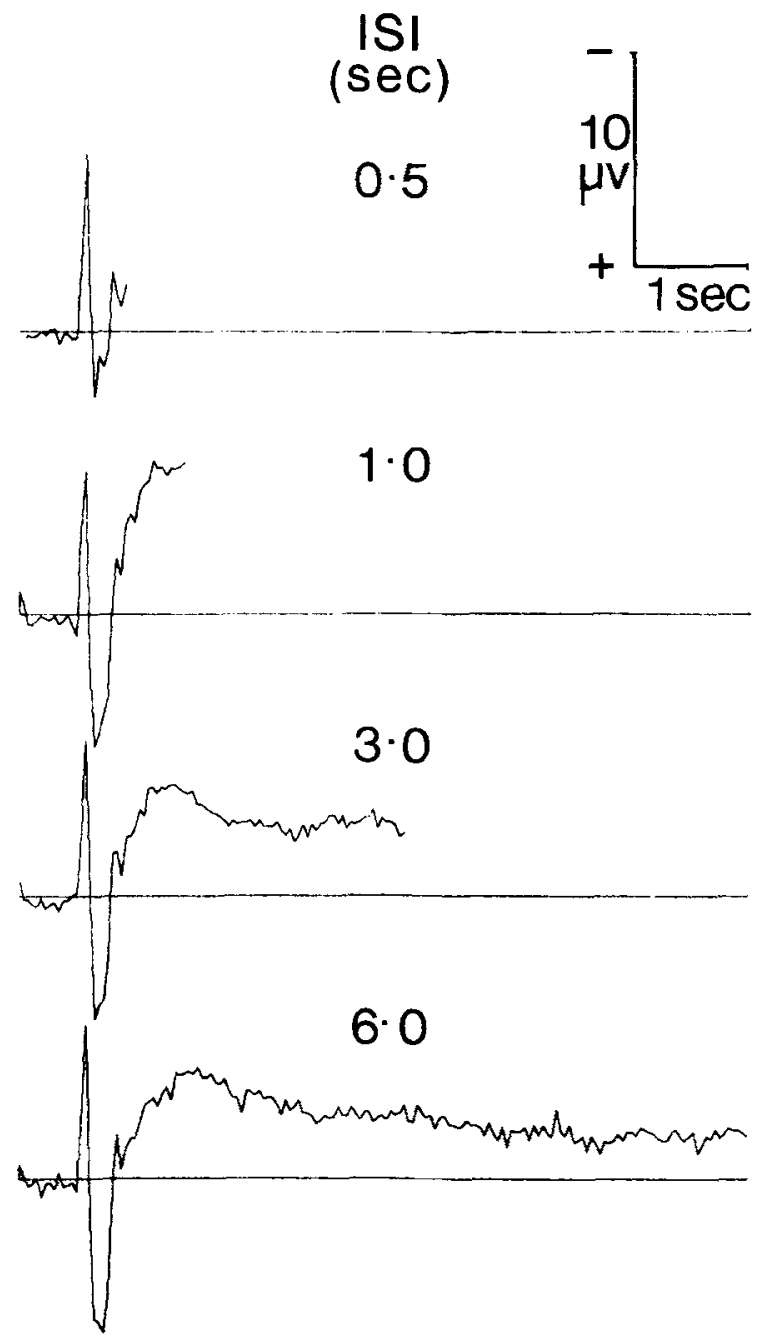

Figure 1. Vertex potentials averaged across subjects as a function of interstimulus interval (ISI); each plot has been terminated at the time of presentation of the critical stimulus. Averages are of 600 trials. 
is contaminated by the blinks which the subjects performed after response as instructed. The morphology of the waveform appears to be consistent across foreperiods. Its time-course, rising to a peak about 1 sec after the warning signal and then slowly declining, is essentially similar to that obtained by Loveless and Sanford (1974a) under irregular foreperiod conditions. Since regular foreperiod conditions were used in the present experiment, it was considered possible that $\mathrm{E}$ waves would develop, as they did in the RT experiment. In fact, upward intlections were observed in the EEGs of some subjects at the termination of some foreperiods, the data shown in Figure 2 being the clearest example of this; but their inconstant manifestation, and small amplitude compared with those obtained in the RT task. makes it doubtful how much significance should be attached to them. Certainly, the absence of any consistent pattern of $E$ waves resolves the methodological difficulty encountered in the design of

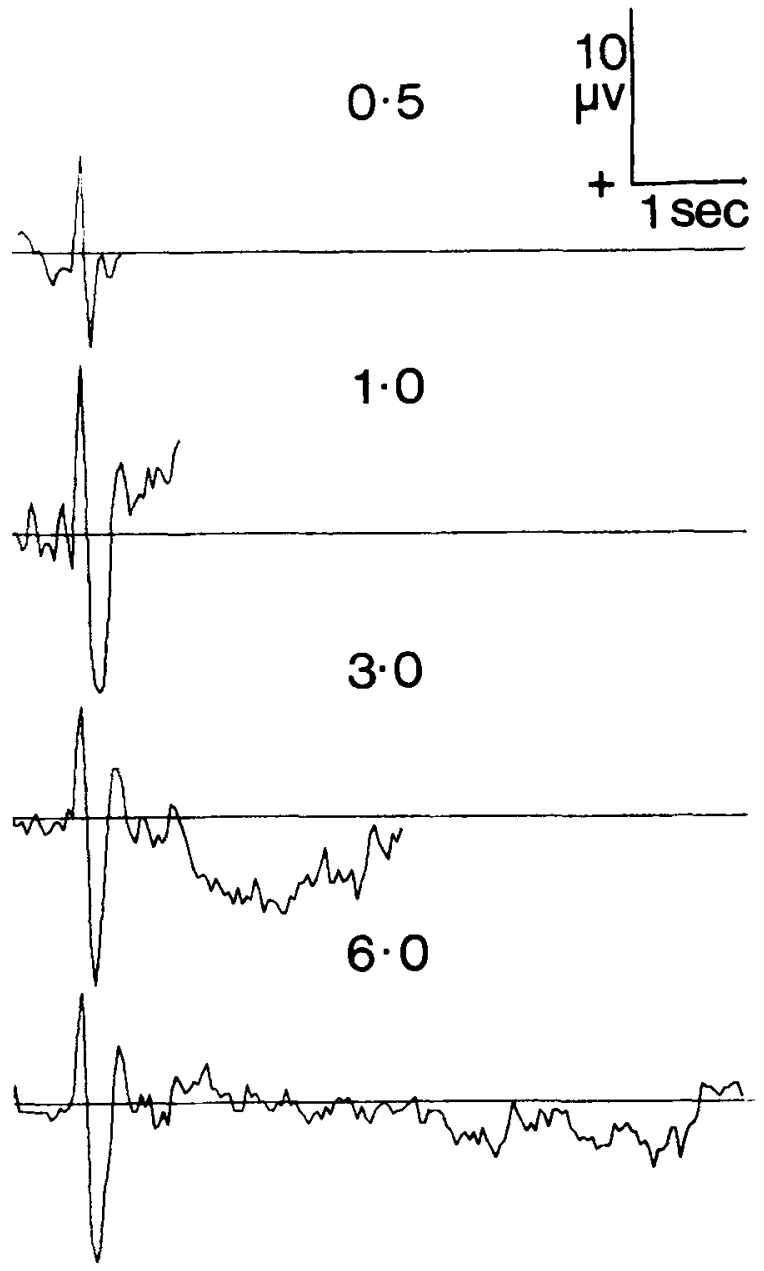

Figure 2. Vertex potentials of one subject as a function of interstimulus interval (ISI); each plot has been terminated at the time of presentation of the critical stimulus. Averages are of $\mathbf{1 0 0}$ trials. this experiment, since the significant change in terminal amplitude of the averaged EEG as a function of foreperiod can contidently be ascribed to the $\mathrm{O}$ ware.

\section{DISCUSSION}

Presentation of a warning signal appears to initiate an increase in sensitivity to subsequent stimuli which is at its maximum during the first second or two, and has substantially declined after $6 \mathrm{sec}$. This finding appears conipatible with functions relating detectability to warning time and interval of time uncertainty obtained by Egan. Greenberg, and Schulman (1961a, b), Egan, Schulman, and Greenberg (1961), and Lowe (1967). Some discrepancy between the time-course of sensitivity and that of the $O$ wave occurs at the 0.5 -sec interval, when the amplitude of the averaged EEG is much lower than at $1.0 \mathrm{sec}$, but the sensitivity difference is comparatively small and nonsignificant. This discrepancy probably results from summation of the initial peak of the $O$ wave with the late positive (P300) component of the evoked potential, so that the measured amplitude appears misleadingly low. With this proviso, the time-course of the $O$ wave mirrors the change in sensitivity rather faithfully. It is therefore reasonable to conclude that this potential expresses the activity of a mechanism regulating sensory gain.

Such a mechanism would account for the effect upon RT of warning signal characteristics which may include modality as well as intensity. Gaillard and Näätänen (1975) compared the effects of auditory and visual warning signals upon the CNV, and found that the auditory signal produced an enhancement of CNV amplitude which was most prominent early in the 1-sec foreperiod, and which therefore seems likely to be attributable to the $O$ wave. Auditory signals produced faster RTs than visual, though not signiticantly so. The authors were uncertain whether the more "arousing" effect of the auditory signal was truly specific to the modality, in view of the difficulty of comparing intensities across modalities. Their results are, in any case, clearly compatible with those of the present experiment.

The same mechanism would appear to account for much of the increase of RT with increasing foreperiod duration, when foreperiods vary over a range comparable to that of the present experiment. However, foreperiod effects in RT tasks may also result from criterion shifts when the subject is able to make some prediction of the time of occurrence of the imperative signal; and the fact that foreperiod duration had no significant effect on criterion measures in the present experiment does not necessarily imply that the subject does not lower his criterion at the end of the foreperiod. Nevertheless. since it has been proposed that criterion changes are 
reflected in the $E$ wave, it is necessary to consider why these waves could not be consistently discerned in the present experiment, as they were in the RT task using the same regular foreperiods (Loveless \& Sanford, 1974a).

A clue may be found in the investigation of the effect of instructions in the simple RT task (Loveless \& Sanford, 1974b), where the $E$ wave was reduced almost to vanishing point under "sensory set" instructions, thus producing a waveform very similar to that obtained in the present signal detection task. The interpretation of this resemblance depends upon the presumed effect of "sensory set" instructions. A number of authors (e.g., John, 1967) have suggested that the real effect of motor and sensory set instructions lies in their implied stress upon the relative importance of speed of response and the avoidance of premature responses. On this view, the common feature of the sensory set and signal detection situations would be that in both cases the stress was on the accuracy of response rather than upon its speed. However, the terms "sensory set" and "motor set" originated in a distinction between concentration upon the occurrence of the imperative stimulus and muscular preparation for the response. Current models of simple RT tend to confuse mechanisms for identifying the stimulus with mechanisms for initiating the response. Thus, John (1967) attributes the slower RT obtained under sensory set to the greater amount of evidence required to decide that the imperative stimulus has occurred, implying that the response released when this decision is taken is equally well prepared under both sets. This is not necessarily true, and it might therefore be argued that the common feature of the sensory set and signal detection situations was a low level of motor readiness. In this case, the $E$ wave might be held to reflect the level of motor preparation rather than the criterion for a cognitive decision. This interpretation may be supported by the observation that the $E$ wave that appears in RT tasks with long foreperiods is morphologically similar to the "readiness potential" which precedes any voluntary movement and exhibits a degree of lateral asymmetry, with greater negativity over contralateral motor cortex (Deecke, Scheid, \& Kornhuber, 1969). Potentials of similar topography associated with motor preparation in warned RT tasks have been reported by Otto and Leifer (1973) and Syndulko and Lindsley (Note 1). These central potentials were dissociated from frontal activity which appears to resemble the orienting response evoked by nonsignal stimuli (Loveless, 1975).

The present evidence is therefore compatible with the view that the $O$ and $E$ waves reflect the activity of topographically distinct generators related to sensitization and motor preparation, respectively. It would not, however, be surprising to find that the configuration of slow potential shifts is more subtly related to the contingencies of various tasks than such a simple dichotomy suggests.

\section{REFERENCE NOTE}

1. Syndulko, K., \& Lindsley, D. B. Motor and sensory determinants of cortical slow potential shifts in humans. Paper presented at the International Symposium on Cerebral Evoked Potentials in Man, Brussels, 1974.

\section{REFERENCES}

Behar, I., \& Adams, C. K. Some properties of the reaction time ready signal. American Journal of Psychology, 1966. 79, 419-426.

Corby, J. C., \& Kopell, B. S. Differential contributions of blinks and vertical eye-movements as artifacts in EEG recording. Psychophysiology, 1972, 9. 640-644.

Deecke, L., Scheid, P., \& Kornhuber, H. H. Distribution of readiness potential, pre-motion positivity, and motor potential of the human cerebral cortex preceding voluntary finger movements. Experimental Brain Research, 1969, 7, 158-168.

Donchin, E., Otto, D., Gerbrandt, L. K., \& Pribram, K. H. While a monkey waits: Electrocortical events recorded during the foreperiod of a reaction time study. Electroencephalography \& Clinical Neurophysiology, 1971, 31, 115-127.

Donchin, E., \& Sutron, S. The "psychological significance" of evoked responses: A comment on Clark, Butler and Rosner. Communications in Behavioral Biology, 1970, 5, 111-114.

Egan, J. P., Greenperg, G. Z., \& Schulman, A. I. Interval of the time uncertainty in auditory detection. Journal of the Acoustical Society of America, 1961, 33, 771-778. (a)

Egan, J. P., Greenberg, G. Z., \& Schulman, A. I. Operating characteristics, signal detectability, and the method of free response. Journal of the Acoustical Society of America, 1961, 33, 993-1007. (b)

Egan, J. P., Schulman, A. I., \& Greenberg, G. Z. Memory for waveform and time uncertainty in auditory detection. Journal of the Acoustical Society of America, 1961, 33, 779-781.

Gaillard, A. W., \& NäÄtänen, R. Modality effects on the contingent negative variation. In W. C. McCallum and J. R. Knott (Eds.), Event-related slow potentials of the brain. Bristol: Wright, 1975.

GRICE, G. R. Stimulus intensity and response evocation. Psychological Review, 1968, 75, 359-373.

Hilly ard, S. A., Souires, K. C., Bauer, J. W., \& Lindsay, P. H. Evoked potential correlates of auditory signal detection Science, 1971, 172, 1357-1360.

Howarth, C. I., \& Treisman, M. The effect of warning interval on the electric phosphene and auditory thresholds. Quarterly Journal of Experimental Psychology, 1958, 10, 130-141.

Howarth, C. I.. \& Treisman, M. Lowering of an auditory threshold by a near threshold warning signal. Quarterly Journal of Experimental Psychology, 1961, 13, 12-18.

JoнN, I. D. A statistical decision theory of simple reaction time. Australian Journal of Psychology, 1967, 19, 27-34.

Loveless, N. E. Distribution of response to non-signal stimuli. In W. C. McCallum and J. R. Knott (Eds.), Event-related slow potentials of the brain. Bristol: Wright, 1975.

Loveless, N. E., \& Sanford, A. J. Effects of age on the contingent negative variation and preparatory set in a reaction-time task. Journal of Gerontology, 1974, 29, 52-63. (a) 
Loveless, N. E., \& SANFord, A. J. Slow potential correlates of preparatory set. Biological Psychology, 1974. 1. 303-314. (b)

Loveless, N. E.. \& SANFord, A. J. The impact of warning signal intensity on reaction time and components of the contingent negative variation. Biological Psychology, 1975, 2 , 185-194.

LowE, G. Interval of time uncertainty in visual detection. Perception \& Psychophysics, 1967, 2, 278-280.

MCGILL, W. J. Stochastic latency mechanisms. In R. D. Luce, R. R. Bush, and E. Galanter (Eds.), Hand book of mathematical psychology. New York: Wiley, 1963. Pp. 309-361.

OTTO, D. A., \& LeIfER, L. J. The effect of modifying response and performance feedback parameters on the CNV in humans. Electroencephalography and Clinical Neurophysiology, 1973. Supplement No. 33. 29.43.

Paul, D. D., \& Sutton, S. Evoked potential correlates of response criterion in auditory signal detection. Science. 1972, 177, 362-364.

Soxolov, E. N. Perception and the conditioned reflex. Oxford: Pergamon Press, 1963.

Sutton, S. The specification of psychological variables in an average evoked potential experiment. In E. Donchin and D. B. Lindsley (Eds.), Average evoked potentials: Methods. results and evaluations. Washington, D.C: U.S. Government Printing Office, 1969. Pp. 237-297.

TECCE. J. J. Contingent negative variation (CNV) and psychological processes in man. Psychological Bulletin, 1972, 77, 73-108.

Treisman, M. The effect of one stimulus on the threshold for another: An application of signal detectability theory. British Journal of Statistical Psychology, 1964, 17, 15-35.

Treisman, M., \& Howarth, C. I. Changes in threshold level produced by a signal preceding or following the threshold stimulus. Quarterly Journal of Experimental Psychology, 1959. 11, 129-142.

W alter, W. G., Cooper, R., Aldridge, V. J., McCallum, W. C., \& WINTER, A. L. Contingent negative variation: An electric sign of sensori-motor association and expectancy in the human brain. Nature, 1964, 203, 380-384.

WeERTS, T. C., \& LANG, P. J. The effects of eye fixation and stimulus and response location on the contingent negative variation (CNV). Biological Psychology, 1973, 1, 1-19.

WINER, B. J. Statistical principles in experimental design. London: McGraw-Hill, 1970.

(Received for publication October 23, 1974; revision received March $10,1975$. ) 